\title{
Experiments on quantum Hall interferometers - towards a measurement of unconventional quantum statistics
}

1. Role of interactions in an electronic Fabry-Perot interferometer operating in the quantum Hall effect regime.

Authors: Nissim Ofek, Aveek Bid, Moty Heiblum, Ady Stern, Vladimir Umansky, and Diana Mahalu, PNAS March 23, 2010 vol. 107 no. 12 5276-5281.

2. Distinct Signatures For Coulomb Blockade and Aharonov-Bohm Interference in Electronic FabryPerot Interferometers,

Authors: Yiming Zhang, D. T. McClure, E. M. Levenson-Falk, C. M. Marcus, L. N. Pfeiffer, K. W. West.

Phys. Rev. B (RC) 79, 241304(R) (2009).

3. Alternation and interchange of e/4 and e/2 period interference oscillations as evidence for filling factor 5/2 non-Abelian quasiparticles

Authors: R.L. Willett, L.N. Pfeiffer, K.W. West

arXiv:0911.0345

\section{Recommended with a Commentary by Ady Stern, Weizmann Institute, Israel.}

If a test of associations was to be run on condensed matter physicists and the associations for the word "topology" were to be asked for, it is likely that the Aharonov-Bohm effect, the quantum Hall effect and anyons would be high on the list of answers. The Aharonov-Bohm effect states that the phase accumulated by an electron encircling a flux tube is determined by the topology of its trajectory. The quantum Hall effect states that the quantized zero temperature Hall conductivity of a two dimensional electronic system is a topological quantity. And the phase associated with one anyon encircling another is determined by the topology of the trajectory. It is then natural to combine these three associations and aim for an experimental demonstration of the anyonic statistics of quantum Hall quasi-particles through interferometry.

The naïve experiment that comes to one's mind is a variation on the Aharonov-Bohm theme: since current in the quantum Hall effect propagates chirally along the edges of a sample, an electronic analog of an optical Fabry-Perot interferometer (See Figure) may be constructed by introducing two 
constrictions into a Hall bar, thus introducing two points of scattering between counter-moving edges on the two sides of the bar. When the constrictions are fairly open, the probability for inter-edge scattering, and thus the longitudinal resistance induced by the constrictions, is an outcome of the interference of two trajectories. When the constrictions get pinched off, multiple reflections play a more prominent role. In both cases, in the regime of the Integer quantum Hall Effect one expects the Aharonov-Bohm effect to lead to oscillations of the resistance of the Hall bar as a function of the flux within the interference loop, with a period of one quantum of magnetic flux. The loop is defined by the two constrictions and the two sample edges. The flux within the constrictions may be varied either by varying the magnetic field or by applying a voltage to a side gate that "moves" the edge of the bar and varies the area of the interferometer. The case of the fractional quantum Hall states is more complicated: in the abelian state the phase accumulated by an interfering quasi-particle should be the sum of two contributions - an Aharonov Bohm phase scaled down by the charge of the quasi-particle, which is a fraction of the electron's charge, and an anyonic phase, the phase accumulated due to the mutual statistics of quantum Hall quasi-particles. A variation of the magnetic field should vary discretely the number of quasi-particles in the interference loop, and the phase jumps associated with these variations are supposed to reflect the abelian fractional statistics of the quasi-particles. Even better, for the putative non-abelian quantum Hall state at $n u=5 / 2$ the variation of the magnetic field should introduce oscillations in the periodicity and amplitude of the interference pattern whenever the number of quasi-particles localized in the interference loop varies from even to odd and vice versa. Altogether, then, the Fabry-Perot quantum Hall interferometer seems to be a promising tool for the experimental study of abelian and non-abelian statistics of quantum Hall quasi-particles. First experiments addressing these issues, by the Goldman and Willett groups, were indeed reviewed here $\wedge 1$.

Naïve theoretical models usually require refinements when experimental results come in, and quantum Hall interferometers are no exception. To start with, observations of interference in the Integer Quantum Hall regime pose some questions. It is convenient to characterize the observed interference in terms of lines of constant phase in the two-parameter plane of gate voltage and magnetic field. For the Aharonov-Bohm (AB) effect one expects that an increase of the magnetic field should be accompanied by a decrease of the loop area for keeping the phase constant. In fact, in most cases the opposite 
behavior is observed. When the magnetic field is increased the gate has to pull the walls of the interference loop outwards to keep the phase unchanged. Furthermore, in these cases the magnetic field period of the interference is not one flux quantum, but rather depends on the identity of the edge state that carries the interfering electrons. In the minority of the observations, particularly in interferometers of large area, the $\mathrm{AB}$ expectations are satisfied by the experiments, and in yet other cases both behaviors co-exist, namely constant phase lines of both types occur in experiments.

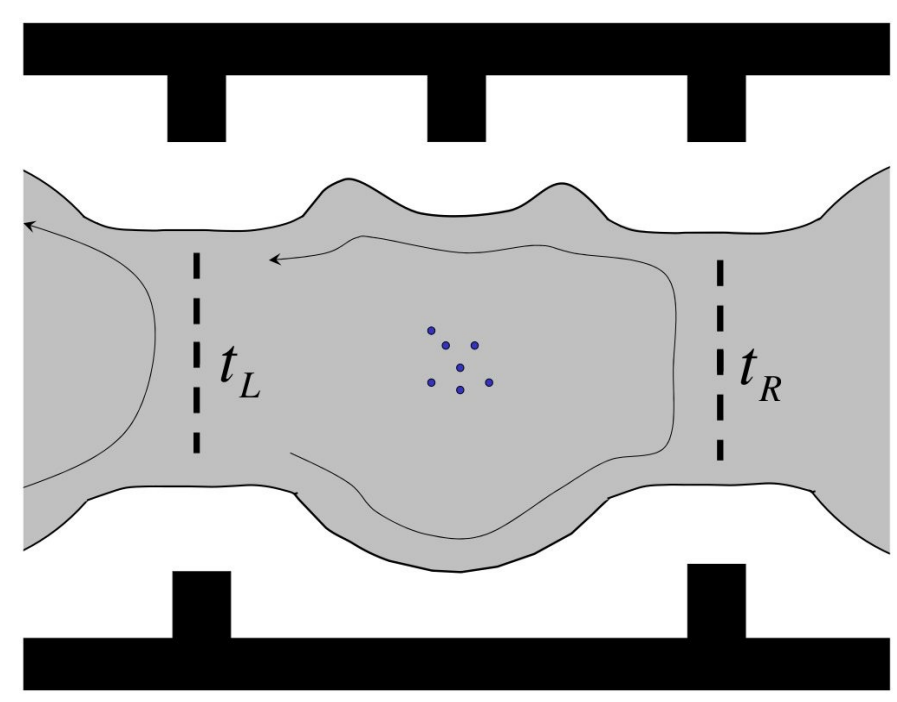

Figure. Fabry-Perot quantum Hall interferometer.

The origin of these observations is likely to be a result of the differences between the experimentally realized quantum Hall Fabry-Perot interferometer and the idealized Aharonov-Bohm setting. Ideally, by varying the magnetic field one varies continuously the flux in the interference loop and discretely the number of quasi-particles in the loop. By varying the gate voltage, one varies continuously the area of the loop and does not affect the number of bulk quasi-particles at all. In real-life interferometers, electron-electron interaction affects this picture in a fundamental way. Since a variation of the magnetic field affects the electronic density, through its effect on Landau levels wave functions and degeneracy, the need to preserve charge neutrality leads to shrinkage of the interference loop with an increasing magnetic field. As a consequence, the flux through the interferometer is not necessarily an increasing function of the magnetic field. Furthermore, the same Coulomb interaction leads also to an effect of the gate voltage on the density at the bulk of the interference loop, and hence on the number of quasi- 
particles in the bulk, in addition to its effect on the area of the interference loop. The regime at which consideration of electron-electron interaction are dominant is commonly referred to as "Coulomb dominated" (CD). The relative position of a particular interferometer on the axis stretching between the $\mathrm{AB}$ and $\mathrm{CD}$ limits depends on sample-dependent parameters as well as on magnetic field, electron density etc., and this dependence is at present not fully understood. It is however easy to experimentally determine the regime at which a particular experiment is.

The experiments reviewed here explore interference in the CD limit. For the integer case, they study the transition to the $\mathrm{AB}$ limit as the area of the device is increased and the interaction between electrons screened. The experiments map the lines of constant phase on the magnetic field-gate voltage plane, both in integer and in fractional states, and show that in the latter case the interfering quasi-particle is fractionally charged. Furthermore, in one of the experiments interference is probed at nu=5/2, with the outcome being an observation of switching of interference periodicity as a function of gate voltage and magnetic field. This switching, reviewed here in the past $^{\wedge} 1$ is suggestive of non-abelian quasi-particles, but is not yet an unambiguous observation.

Do the effects of electron-electron interaction affect the extent to which these interferometers may be used for an experimental probe of fractional statistics in the quantum Hall effect? The fractional statistics has a signature in determining the resistance of the interferometer, and the question is only whether this signature can be separated from other effects, in particular the effects of area variation due to electron-electron interaction in the $\mathrm{CD}$ limit. While in principle the effects of quantum statistics may be separated from the Coulomb effects both for abelian and non-abelian states, this separation is expected to be much easier for the non-abelian case. For this case, the amplitude and periodicity of the interference are determined by the parity of the number of quasi-particles in the bulk, and are unaffected by the area of the interference loop. The effects of non-abelian statistics should then be easy to separate even in the extreme CD limit. In contrast, for abelian states the statistics manifests itself in the phase of the interference pattern, which is affected also by the area of the loop. For both types of states, we expect that as further experiments and theory allow us better understanding and control of the position of a particular experiment on the AB-CD axis, the interferometers will realize their potential of being "statisticometers".

1. S. Kivelson and C.Nayak, http://condmatjournalclub.org May 2009). 